\title{
Prototyping adaptive systems in smart environments using virtual reality
}

\author{
Francesca Gullà ${ }^{1}$. Roberto Menghi ${ }^{1} \cdot$ Alessandra Papetti $^{1} \cdot$ Marina Carulli $^{2} \cdot$ Monica Bordegoni $^{2}$. \\ Andrea Gaggioli $^{3}$. Michele Germani ${ }^{1}$
}

Received: 30 October 2018 / Accepted: 6 December 2018 / Published online: 18 December 2018

(c) Springer-Verlag France SAS, part of Springer Nature 2018

\begin{abstract}
Smart environment is a key challenge for current ICT research: it is one of the solutions that can enhance people's quality of life and enable users with impairment to live independently. Over the years, scientific research has proposed several solutions to help and improve the capabilities of its occupants, but they are often developed for a specific context (e.g. particular disease or impairment). These systems do not adapt to the real needs of users with different profiles, and neglect that the user's requirements may evolve over time. This research work aims to develop a new adaptive smart system able to support users (with and without disabilities) in performing daily tasks by recognizing their preferences and actions and adapting the system feedback consequently. With the aim to develop an easy, efficient and usable adaptive smart system, the final users have been involved in the whole design and development process. The system was validated through a virtual reality system allowing the user interaction evaluation and helping the usability improvement.
\end{abstract}

Keywords Smart environment - Adaptive and adaptable user interface · Virtual reality system · Bayesian network · ICT · User-centered design

\section{Introduction}

In recent decades, people's daily lives have been transformed by the faster developing of information and communica-

Francesca Gullà

f.gulla@univpm.it

Roberto Menghi

r.menghi@univpm.it

Alessandra Papetti

a.papetti@univpm.it

Marina Carulli

marina.carulli@polimi.it

Monica Bordegoni

monica.bordegoni@polimi.it

Andrea Gaggioli

andrea.gaggioli@unicatt.it

Michele Germani

m.germani@univpm.it

1 Università Politecnica delle Marche, Via Brecce Bianche, Ancona, Italy

2 Politecnico di Milano, Via La Masa, Milan, Italy

3 Università Cattolica di Milano, Largo Gemelli, Milan, Italy tion technologies (ICT). In particular, the spreading of smart products and the possibilities to connect IoT devices together have changed the living environments. Cook and Das defined these spaces as Smart Environments: "a small world where different kinds of smart device are continuously working to make inhabitants' lives more comfortable" [1]. Smart environment is a key challenge for current ICT research: it is one of the solutions that can improve people's quality of life and enable users with impairment to live independently.

The World Health Organization has estimated that around $10 \%$ of the world's population (i.e. 650 million) live with a disability and the percentage is increasing due to population growth, medical advances and the ageing society [2]. In Asia, North America and Europe, the percentage of people aged 65 or older is projected to grow and it is estimated that by 2030 it will be between 12 and 24\% [3]. In addition, there will be a rapid increase in number of people with mental and physical impairments as well as various age-related chronic diseases such as hyperactivity disorder, autism spectrum disorders, motor handicap and cognitive decline [4]. They have specific needs and restrictions caused either directly by ageing or any diseases that change over 
time and affect their daily domestic activities. The main difficulties are turning electrical or gas-driven appliances on and off, preparing meals, managing household appliances, etc. Smart environments have the potential to support the independent lives of people with impairment, since they can improve comfort and energy efficiency, provide a continuous monitoring, support them in performing daily life activities, provide a safe environment and help individual rehabilitation [5]. They provide additional supportive functions by collecting environmental data and improving the user experience in that environment.

Over the years, scientific research has proposed several examples that help and improve the capabilities of its occupants, particularly in performing domestic tasks. However, most of these case studies focused often only on provided services and do not take full account of end user preferences and applications. Experimentations are driven by technology improvement (technology push) and not from a careful user's needs analysis. Several solutions have been proposed (e.g. to support users in performing daily life activities [6], preparing meals [7], controlling household appliances [8, 9]), but they obtained rather low experienced benefits. The main reason was the lack of adaptation, as the system did not adapt to the real needs of users with different profiles.

This research work aims to develop a new adaptive smart system able to support any user in performing cooking activities and to help in carrying out domestic tasks that might otherwise be difficult or impossible. The system has been designed for normal people as well as users with several kinds of impairment (i.e. visual, motor, cognitive) in according with Design for All approach. It allows managing all devices and appliances improving the usability for various utilization profiles/contexts and for different user requirements/skills. The system supports the user in the daily tasks execution by recognizing his/her preferences and actions and adapting the system feedback consequently. It has been designed following an interactive engineering design approach that takes into account the expectations and constraints of stakeholders (i.e. people with impairments, relatives and caregivers) and through collaborative and interactive methods. Indeed, the final users have been involved in the whole design and development process, according with ISO 9241-210:2010 [10] with the aim to develop an easy (self-explanatory), efficient and pleasant (usable) adaptive smart system. A team of five experts (i.e. two geriatricians, two psychologists and an expert in human computer interaction) was also engaged in the research work in order to enhance the user-centered and collaborative design process. Finally, the system was validated through a Virtual Reality (VR) system allowing the user interaction evaluation and helping the usability improvement $[11,12]$.

\section{Research background}

Adaptive systems are entities that adapt their displays and available actions to the user's current goals and abilities, by monitoring user status, the system state and the current situation [13]. Benyon and Murray [14] defined Adaptive Systems as "systems which can automatically alter aspects of their functionalities and/or interface in order to accommodate the differing needs of individuals or groups of users and the changing user needs over time". To achieve this, such systems have to learn the context in which they evolve and to adapt their interfaces and interaction modalities to communicate with users.

The research literature describes many approaches that can be used to design flexible user interfaces, which can be classified into two broad categories: adaptable and adaptive. Adaptable User Interfaces (AdUIs) can be defined as systems in which the activation of user-computer interaction is performed by the final user through the selection of a specific user profile from a predefined list. In an adaptable interface the interface features can be modified by the user to fit the context of use [15]. The Adaptability is based on the users' known characteristics and preferences; these are defined prior to their interaction session and, in any case, are assumed to remain static during the session [16]. Instead, Adaptive user interfaces (AUIs) are systems that change its structure, contents and elements according to the need and context of the user $[17,18]$. An adaptive interface autonomously adapts its information processing mechanisms and their actions to meet current goals and abilities of the user [13]. AUIs are situated and aim to enable the user to become smarter and more empowered. Adaptive user interfaces may be considered as a special class of learning systems that are developed to aid humans, in contrast with traditional ones, which was introduced with the aims to replace domain experts [19].

The separation between the adaptivity and adaptability is very thin. Both approaches have their pros and cons. The most important advantage of adaptable systems is that the users are in total control of the individual appearance and interface [20]. Otherwise, the use of adaptive user interface seems to help to improve user interaction with systems by facilitating user performance, minimizing the need to request help, easing system use, helping users deal with complex systems and avoiding cognitive over-load problems. However, the changes made by the UI can disorient the final user [15, 20]. In spite of AUI research progress, there are many articles and books that focus on restrict domains since researchers come from diverse scientific disciplines. Furthermore, no technique has been shown to be applicable at a general level.

The literature on the adaptive interfaces research context is very heterogeneous and closely linked with each domain of application. There are several contexts in which the adaptive systems have been used: health, smart environments, 
air traffic control, web applications, electrical network management and thermal plant supervision, warehouse picking system, web services, mobile device context etc.

In the health context, for example, the adaptive user interface has been used in a hypermedia data browsing to develop a clinical workstation prototype for the cardiology domain: "HEMA". In detail, it has been designed for the reviewing of patients record [21]. On the basis of the user's type, two HEMA prototypes display has been provided: the system automatically displays the interface model associated to the group's profile. Moreover, Bental et al. [22] provided a framework for the provision of computer-based information for cancer patients through the interactive system developing, which provide at the right time health information to the patient. Shakshuki et al. [23] provided a multi-agent system for tracking and monitoring health data for patients through a system architecture developing. Two scenarios have been used to test the system: patient and doctor use. Finally, the adaptive user interfaces have been used to develop a framework for eHealth [24].

In the smart environments context, the adaptive user interface has been used to manage and control various devices simultaneously in a modern home theater system including smart TV, DVD etc. For example, Song and Cho [25] modelled the ubiquitous home environment through the use of a Bayesian network able to predict the custom devices in each situation. Furthermore, Namgoong et al. [26] proposed an adaptive interface to enable end user to utilize the devices easily, through the semantic descriptions and ontology specification.

Yen et al. provided a generalized framework for the UI adaptation in complex environments. In particular, the framework has been tested in a complex supervisory task: Air Traffic Control. In order to test the interface a data set (time, number of mouse clicks etc.) for each interface has been collected [27].

El-Bakry et al. presented a new adaptive interface design for web application. In particular, they described guidelines, architecture, languages and framework for the web application interface design [28]. Moreover, Rebai used a Bayesian Networks to design an adaptive Social Network interface for web applications. To test the new approach the web interface that allows exchanging documents and courses between students and teachers has been tested [29].

In the Electrical Network Management field and in the Thermal Power Plant supervision an innovative autoadaptive multimedia interface (AAMI) architecture has been developed and implemented. The benefits from the AAMI approach has been evaluated [30].

In addition, the adaptive user interfaces have been used in a work smart environments context, for a warehouse picking system [31]. In particular, they exploited the model-based approach in supporting adaptation and developed a set of rules to provide the adaptation action. In a supermarket chain the system has been tested, through the GUI Application. A user study has been conducted to evaluate the rules from the end user point of view.

In the mobile device context, the adaptive user interface has been used to adapt a graphical interface to a various type of user [32-34].

In this context, this paper aims to enrich the state of the art by proposing a new application of adaptive systems in the smart home. The main innovative aspects are the following: (1) the effective combination of adaptive and adaptable elements; (2) the adoption of a Design for All approach, which allows improving the user experience of people with and without disabilities and (3) the adoption of an users-centered approach that means fitting the system to the users needs and involving them in the design process.

Although there exist today a number of established approaches and frameworks for the design and implementation of AUIs, their evaluation is yet to be addressed in a comprehensive way. In fact, there are no unique valuation methods in this field that provide adequate feedback in the AUI design process. Once of the most used popular technique is the comparison between adaptive and non-adaptive system in terms of user satisfaction [35-37]; adaptive support effect [38, 39]; user performances [40], usability [41] and efficiency [42]. Another approach in the AUI evaluation has been put forward by Paramythis et al. [43] that provide a method to separately evaluate each adaptive system constituents through the decomposition of adaptation "from the perspective of HCI-oriented evaluation": adaptation is no longer considered as a singular process. Other approaches concern the adaptive interface evaluation in terms of cost and benefit [44] user's error [45], cognitive workload predictability and accuracy [46], and accessibility [47].

An emerging trend is also the application of self-adaptive methods within virtual environment (VE). Adaptation is one of the five core technologies in VR-based training, which are arranged within a variety of topics: medical, industrial and commercial, collaborative VR and MOOCs, serious Games and rehabilitation [48]. The most common is the medical one. For example, it has recently been widely used in the field of augmented stroke rehabilitation. Huang et al. [49] in their study, proposed the combination of force-rendered AAN adaptive control, VR8-based RGS, and transferring VE with the aim of optimizing the training effectiveness and enhancing the rehabilitation efficiency, conducting clinical trials on achronic stroke patient.

In the industry sector, it has been demonstrated how a VE can be used for trying out safe human-robot collaboration techniques: proactive and adaptive [50]. In this case, the $\mathrm{VE}$ is used to validate the system before its implementation in real robotic cells. Furthermore, the VE is used to evaluate the end-user experience and satisfaction while using 
Table 1 System functions related to the oven

\begin{tabular}{|c|c|c|c|}
\hline Household appliance & Function & User $\rightarrow$ system & System $\rightarrow$ user \\
\hline \multirow[t]{15}{*}{ Oven } & On-off & Turn the oven on/off & Feedback on machine status \\
\hline & \multirow[t]{3}{*}{ Cooking program setting } & $\begin{array}{l}\text { Select the cooking program from } \\
\text { the list }\end{array}$ & Feedback on selected cooking program \\
\hline & & Set the cooking temperature & Feedback on set cooking temperature \\
\hline & & Set the cooking time & Feedback on set cooking time \\
\hline & \multirow[t]{2}{*}{ Start/stop } & Start/stops the cooking & Feedback on cooking status \\
\hline & & Schedule the cooking start time & Feedback on scheduled start time \\
\hline & \multirow[t]{3}{*}{ Cooking monitoring } & \multirow[t]{3}{*}{ Monitor the cooking } & Feedback on cooking progress \\
\hline & & & Feedback on set temperature \\
\hline & & & Feedback on cooking time (if it has been set) \\
\hline & \multirow[t]{4}{*}{ Automatic setting } & \multirow[t]{4}{*}{ Select the recipe } & Set the cooking program according to the recipe \\
\hline & & & Feedback on cooking program \\
\hline & & & Feedback on cooking temperature \\
\hline & & & Feedback on cooking time \\
\hline & \multirow[t]{2}{*}{ Instructions } & Ask for an information & Link to the household appliance manual \\
\hline & & Ask for a support & $\begin{array}{l}\text { Activate an interactive guide that supports the } \\
\text { user step-by-step }\end{array}$ \\
\hline
\end{tabular}

different tool [51]. A similar approach can be exploited to preliminary test adaptive domestic systems, but no work has been reported in the literature. Experimenting the proposed adaptive framework through VR, this paper would be a first attempt to investigate this issue.

\section{The system adaptive framework}

The main goal of this research work is the development of an adaptive system that enriches the user interaction with a daily life environment, the kitchen. It has to support the user in the appliances management (i.e. fridge, oven, dishwasher) and the daily tasks execution (e.g. meal preparation) by recognizing his/her preferences, analyzing his/her actions and adapting the system feedback consequently. In this section, the definition of its architecture is described.

In order to support the design phase, a reliable and realistic representation of target user groups for reference has been created. It is based on ethnographic analysis. It allowed giving a clear picture of the users in terms of needs and expectations, from their backgrounds, limits, goals, and values to how they are likely to interact with the system. In particular, the personas $[52,53]$ related to the following three clusters of users have been defined: (U1) subjects with cognitive impairment, (U2) subjects with dexterity problems and (U3) visually impaired.

Personas helped to uncover universal features and focus decisions surrounding system components by adding a layer of real-world consideration. It led the definition of system functions, of which an extract is given in Table 1 . They refer to a kitchen environment that implements a home automation system able to detect and learn the user's behavior and help him/her accordingly, through an adaptive user interface.

In order to define the adaptable and adaptive elements of the system, several use cases have been defined. As example, that concerning the cooking with the oven is shown in Table 2.

A system architecture able to satisfy the user groups expectations and implement the desired functions has been developed (Fig. 1). It consists of three main modules, which are described in the following sections: Database Management System (DBMS), Core, and Interface. The user-system interaction, which takes place by a mobile device (e.g. tablet), is recognized by the system in terms of user profile and action. It occurs through a constant communication between Core and DBMS, which also allows increasing the network knowledge and managing the adaptation. As a consequence, the Interface content and graphics elements are subject to change [54].

\subsection{Database}

The DBMS is a software system aimed to store the user and context information, collect all the data deriving from different system inputs and enable an efficient querying.

In details, data are structured in four semantic areas:

- User Profile, which comprises the user information such as demographic attributes, attitude, personal skills, healthrelated attributes, etc.; 
Table 2 Oven use case definition

\begin{tabular}{|c|c|}
\hline \multicolumn{2}{|c|}{ Use case: Cooking something with the oven } \\
\hline \multicolumn{2}{|c|}{$\begin{array}{l}\text { The user turns on the oven and verifies that it is connected to the network. He/she interacts with the } \\
\text { interface to set the cooking program. He/she starts the cooking and monitors it until completion. } \\
\text { Lastly, he/she turns off the oven }\end{array}$} \\
\hline \multicolumn{2}{|c|}{ Involved functions } \\
\hline \multicolumn{2}{|l|}{ On-off } \\
\hline \multicolumn{2}{|l|}{ Cooking program setting } \\
\hline \multicolumn{2}{|l|}{ Start/stop } \\
\hline \multicolumn{2}{|l|}{ Cooking monitoring } \\
\hline \multicolumn{2}{|l|}{ Adaptable elements } \\
\hline On/off command & $\begin{array}{l}\text { Depending on the user profile, different types of interaction are } \\
\text { enabled (e.g. tap, swipe, voice recognition) }\end{array}$ \\
\hline Cooking program selection & $\begin{array}{l}\text { Depending on the user profile, different types of interaction are } \\
\text { enabled (e.g. tap, swipe, voice recognition) }\end{array}$ \\
\hline Cooking temperature setting & $\begin{array}{l}\text { Depending on the user profile, different types of interaction are } \\
\text { enabled (e.g. tap, swipe, voice recognition) }\end{array}$ \\
\hline Cooking time setting & $\begin{array}{l}\text { Depending on the user profile, different types of interaction are } \\
\text { enabled (e.g. tap, swipe, voice recognition) }\end{array}$ \\
\hline Cooking start time scheduling & $\begin{array}{l}\text { Depending on the user profile, different types of interaction are } \\
\text { enabled (e.g. tap, swipe, voice recognition) }\end{array}$ \\
\hline Start/stop command & $\begin{array}{l}\text { Depending on the user profile, different types of interaction are } \\
\text { enabled (e.g. tap, swipe, voice recognition) }\end{array}$ \\
\hline $\begin{array}{l}\text { System feedback on machine status } \\
\text { and cooking program setting }\end{array}$ & $\begin{array}{l}\text { Depending on the user profile, the feedback is provided through } \\
\text { different sensory channels (e.g. visual, acoustic) }\end{array}$ \\
\hline \multicolumn{2}{|l|}{ Adaptive elements } \\
\hline Cooking program selection & $\begin{array}{l}\text { According to the user's profile and his/her previous interactions, } \\
\text { the system suggests the cooking program by highlighting it, } \\
\text { setting it as default, or in any case facilitating its selection }\end{array}$ \\
\hline Cooking temperature setting & $\begin{array}{l}\text { According to the user's profile and his/her previous interactions, } \\
\text { the system suggests the cooking temperature by highlighting } \\
\text { it, setting it as default, or in any case facilitating its setting }\end{array}$ \\
\hline Cooking time setting & $\begin{array}{l}\text { According to the user's profile and his/her previous interactions, } \\
\text { the system suggests the cooking time by highlighting it, } \\
\text { setting it as default, or in any case facilitating its setting }\end{array}$ \\
\hline Cooking start time scheduling & $\begin{array}{l}\text { According to the user's profile and his/her previous interactions, } \\
\text { the system suggests the delay start by highlighting it, setting } \\
\text { it as default, or in any case facilitating its setting }\end{array}$ \\
\hline
\end{tabular}

- User-System Interactions, which includes all the data related to the interaction with the environment and the use of the household appliances (e.g. selected options, preferences, switch on/off, etc.);

- Adaptation Actions, which contains the log of all the adaptations carried out by the system (e.g. adaptation of program default, adaptation of temperature default, etc.). Whenever the system performs an adaptation, the related action is stored in the database in order to monitor the system adaptation rate and, consequently, improve the user experience;

- Context, which refers to all the relevant information for the context definition. It includes the data related to devices (e.g. status, set program, etc.) and timing (e.g. day, time, etc.).

\subsection{Core}

The brain of the application is represented by the "core" layer that provides the application logic management. The Core is composed of two engine mechanisms (Adaptable and Adaptive) and a monitoring system of changes.

The Adaptable Engine shall make the system more suitable to the user profile. Adaptability is based on features and preferences that are known at a first interaction, and they are assumed to remain static during a single session of interaction. This engine takes as input the collected information in the User Profile to adapt the graphical interface features, such as layout, font and zoom. The technical accessibility requirements and the corresponding control points for compliance verification have been defined according to the standards, guidelines and success criteria contained in 


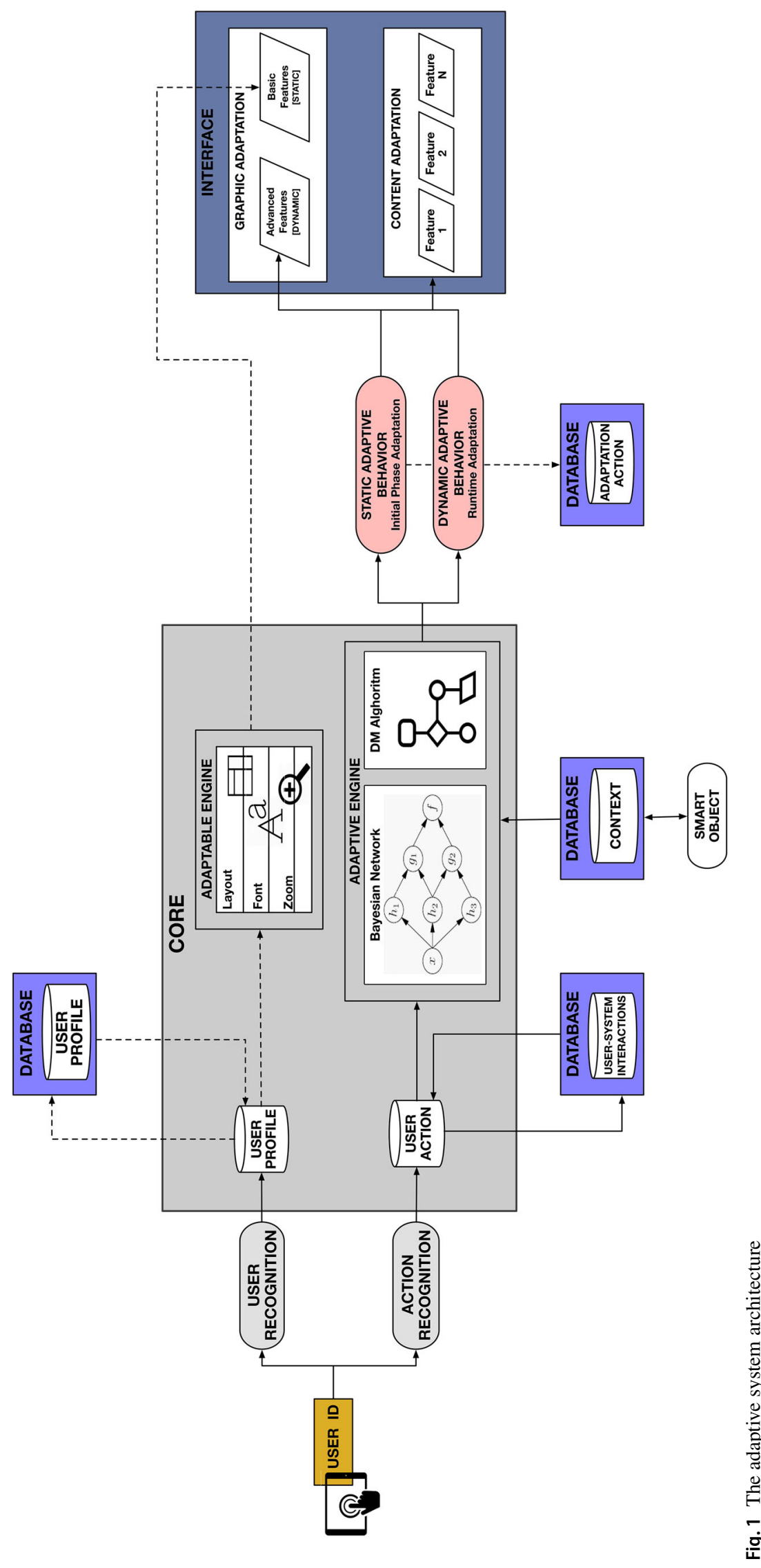


the recommendation that the World Wide Web Consortium (W3C) - Web Accessibility Initiative (WAI) published on 11 December 2008 and which contains the Web Content Accessibility Guidelines 2.0 (WCAG 2.0) [55].

The Adaptive Engine represents overall adaptive system pivot: it is composed of an adaptive mechanism and a decision-making algorithm (DMA). It makes the system adaptive according to the user profile. In particular, adaptivity consists of change mechanisms, which include all dynamic features (i.e. preferences, information contents, graphic elements, etc.), based on user's interactions history. The adaptive mechanism is based on the Bayesian Belief Networks (BBNs) that are one of the most comprehensive tools and more consistent for the acquisition, representation and exploitation of knowledge in conditions of uncertainty $[56,57]$. Moreover, a DMA is defined to manage the correct adaptation behavior according to the user action.

As shown in Fig. 1, the user actions are recognized through the Action Recognition module, locally stored in the "UserSystem Interactions" database, and sent to the Adaptive Engine module, which updates the BBNs and provides two types of outputs:

- Static adaptive behavior This output sets the type of interface and initial home appliance defaults. It takes care of the initial interface setup configuration and the initial default. It is handled by calculating the probabilities associated with finding the User System Interaction, and will set the defaults as the last adaptation made (the previous state, ended);

- Dynamic adaptive behavior This output refers to user actions (which are saved and given to the network at run time). It also manages household appliance defaults and updates the interface at run time.

\subsubsection{Bayesian network}

The Bayesian network has been designed by assuming the use of the system in a home environment, specifically in the kitchen environment, featuring mainly three household appliances: oven, dishwasher and refrigerator. In order to manage BBNs, the use of the simulation software Netica, which is produced by Norsys Software Corporation (Netica Application) is proposed.

The network, consisting of a total of $\mathbf{2 5}$ knots (Fig. 2), is structured as follow:

1. Season Consisting of four periods of the year (Autumn, Winter, Spring, Summer)

2. Week Consisting of seven days of the week (Monday, Tuesday, Thursday, Friday, Saturday, Sunday)
3. Day_Range Consisting of four time bands of the day (Range $1=06.01-12.00$, Range2 $=12.01-18.00$, Range $3=18.01-0.00$, Range $4=0.01-6.00$ )

4. Household_Devices Consisting of three appliances (Oven, Dishwasher, Fridge)

5. O_Information_Modality Consisting of the GUI oven menu display modes ("Normal" or "Wizard")

6. O_CycleTarget Consisting of the oven programs list (defrosting, leavened, food_warmer, static, airy, thermoventilated, grill, cleaning)

7. Help_level_O_cycleTarget Consisting of the program list instruction manual for the oven ("Basic" or "Advanced")

8. O_CyclePeriod Consisting of oven cooking times in minutes $($ Range $0=5-25$, Range $1=30-60$, Range $2=$ $65-120$, Range $3=125-180$, Range $4=185-240$ )

9. Help_level_O_cyclePeriod Consisting of the cycle period instruction manual for the oven ("Basic" or "Advanced")

10. O_TemperatureTarget Consisting of the oven temperature list (Range $0=20^{\circ}-35^{\circ}$, Range $1=40^{\circ}-65^{\circ}$, Range $2=70^{\circ}-120^{\circ}$, Range $3=130^{\circ}-160^{\circ}$, Range $4=$ $170^{\circ}-220^{\circ}$, Range $5=230^{\circ}-250^{\circ}$ )

11. O_StartTime Consisting of the time of the day on which the delayed start of the oven is set $(\operatorname{Range} 0=0.01-1.00$, Range $1=1.01-2.00$, Range $=2.01-3.00$, Range $3=$ $3.01-4.00, \quad$ Range $4=4.01-5.00, \ldots, \quad$ Range $23=$ 23.01-0.00)

12. Help_level_O_startTime Consisting of the delay start instruction manual for the oven ("Basic" or "Advanced")

13. TemperatureFreezer Consisting of the freezer temperature list $\left(-18 /-10^{\circ} \mathrm{C}\right)$

14. TemperatureFridge Consisting of the fridge temperature list $\left(0-7{ }^{\circ} \mathrm{C}\right)$

15. F_Information_Modality Consisting of the GUI fridge menu display modes ("Normal" or "Wizard")

16. D_Information_Modality Consisting of the GUI dishwasher menu display modes ("Normal" or "Wizard")

17. D_CycleTarget Consisting of the dishwasher programs list (pre_wash, fragile, eco, rapid, daily, intensive)

18. D_CycleSetting Consists of dishwasher wash options (Top, Bottom, Both)

19. Help_level_D_cycleTarget Consisting of the program list instruction manual for the dishwasher ("Basic" or "Advanced")

20. D_CycleSoap Consisting of washing options related to the presence/absence of detergent

21. D_StartTime Consisting of the time of the day on which the delayed start of the dishwasher is set (Range $0=0.01-1.00, \quad$ Range $1=1.01-2.00$, Range $2=2.01-3.00$, Range $3=3.01-4.00$, Range $4=$ $4.01-5.00, \ldots$, Range $23=23.01-0.00$ ) 


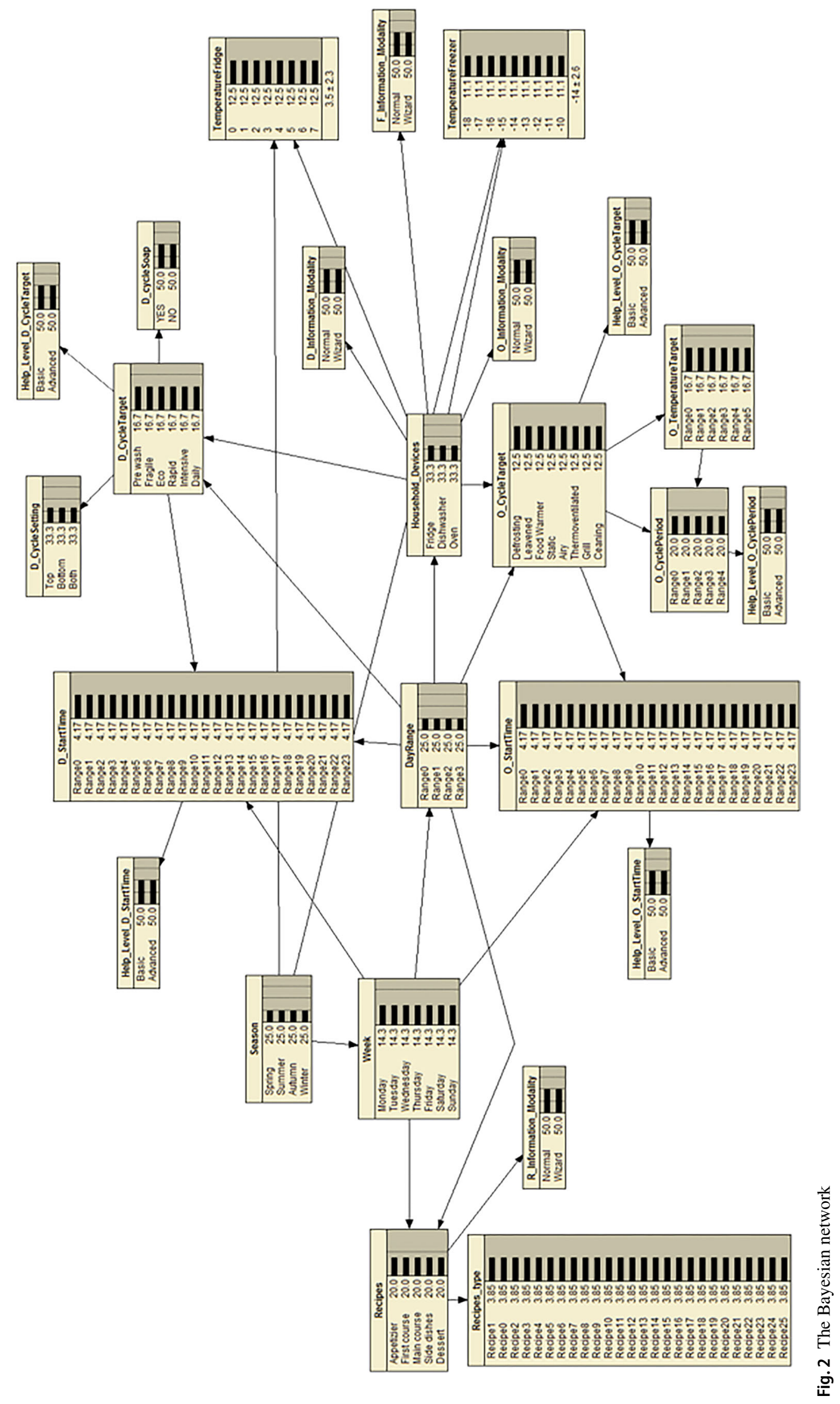


22. Help_level_D_startTime Consisting of the delay start instruction manual for the dishwasher ("Basic" or "Advanced")

23. Recipes Consisting of recipes (Appetizier, First_course, Main_course, Side_dishes, Dessert)

24. R_Information_Modality Consisting of the GUI recipe menu display modes ("Normal" or "Wizard")

25. Recipes_Type Consisting of recipes in the database (Recipe 1-5: Appetizier, Recipe 6-10: FirstCourse, Recipes 11-15: MainCourse, Recipes 15-17: SideDishes, Recipes 17-20: Dessert).

\subsubsection{Decision making}

In order to detect the appropriate system adaptive behavior, a DMA has been used [58]. In particular, the DMA supports the identification of proper system adaptation actions, according to collected user and context data. It is mainly based on two parameters that verify the existence of a predominant probability for a specific event (e.g. user's action or goal) and trigger system adaptive action only in this case.

The first one is $\alpha$ that describes the gap between the probabilities and provides a first level dominance estimation. The latest is $\rho$ that measures the standard deviation of the residual probability. In order to define an algorithm able to provide in output a boolean condition to inform the system about the right adaptation action two thresholds for $\alpha$ and $\rho$ has been defined: respectively called $\alpha$ th and $\rho$ th. The adaptation event is triggered, if both the conditions $\alpha>\alpha$ th and $\rho>\rho$ th are verified.

The thresholds change according to the network nodes number. In this case, to allow the network adaptation and to avoid gross and ineffective behaviors, the following threshold values has been defined: $\alpha$ th $=0.14$ and $\rho$ th $=0.03$. In order to test the network running and its adaptation, a simulation tests has been carried out.

\subsubsection{Simulation}

On the basis of the user profile and its interactions with the system, a trained network has been associated with each subject (U1, U2, U3). The network learning lasted about 1 month. As example, the trained network for the $\mathrm{U} 3$ profile is shown in Fig. 3; the user prefers the oven and dishwasher appliances in the range from 18.00 to 00.00 p.m., in the Normal mode. In particular, for the oven setting, the user prefers the static program at a temperature of $230-250{ }^{\circ} \mathrm{C}$, while for the dishwasher setting it prefers the Rapid program and the "Top" cycle setting.

Once learning the system, the simulation test was performed. In detail, by analyzing the probabilities existing between the nodes characterizing the BBN, it can be seen how the $\alpha$ th and $\rho$ th set for the DM algorithm manage the correct adaptation action. Indeed, as can be seen in Fig. 4, two are the simulation output: the "No Filtered" and the "Filtered" nodes. In particular, the unfiltered knots are represented by probabilities that do not satisfy the specific condition $(\alpha>\alpha$ th and $\rho>\rho$ th) such as week, recipes, O_start_time, etc., and do not suggest the adaptation action. Instead, the Filtered nodes are represented by probabilities that satisfy the specific conditions and suggest the right adaptation action (household_devices, O_temperature_target, etc.).

\subsection{Interface}

The user interface is the module between the system and the end users and it supports them in preparing meals and controlling smart home devices. It is managed by the Application Core that modifies its features so as to make easier and more efficient the domestic tasks. The adaptation mechanism is based on recognition of the user's characteristics and actions, and it can operate both on the graphic features and on the contents of the interface. With the aim to support the greater number of end user, two different UI modalities were designed (Fig. 5): Normal for users without cognitive dysfunction and characterized by a good technology attitude and Wizard for users who have not familiarity with technology and/or have some cognitive dysfunction.

The first one is device-oriented, and it is designed to optimize the user interaction: through the UI, users are able to configure all device settings in the order he/she prefers (e.g. program, temperature, duration and time in the oven setting). The second is task-oriented and it is designed to simplify the user interaction: tasks were decomposed into more simple and basic pre-programmed actions according to a specific and preset order and the system finishes each action after asking for users' confirmation. In this modality, the user must not manage and understand all the information of the appliance or a recipe's procedure: the system tries to make up for the lack of some user ability and it helps him/her to accomplish the activity of daily living independently.

To improve the usability, three different modes of interaction have been integrated into the interface: tap, swipe and drag. Based on user preferences acquired by the system in the profile phase acquisition, the system will provide a specific interaction mode or a combination of these (i.e. tap and insertion, tap and swipe, tap and drag). In addition, in the functional model the system adaptation rules have been inserted. They determine the system response according to user actions and number of interactions. In accordance with the standards and the design guidelines $[10,59]$ the GUI has been designed for a touch screen application to provide high accessibility and usability for all kinds of users.

The adaptation mechanism changes both the graphic features and the contents of the interface. The graphic features have been designed to compensate any possible user's impair- 


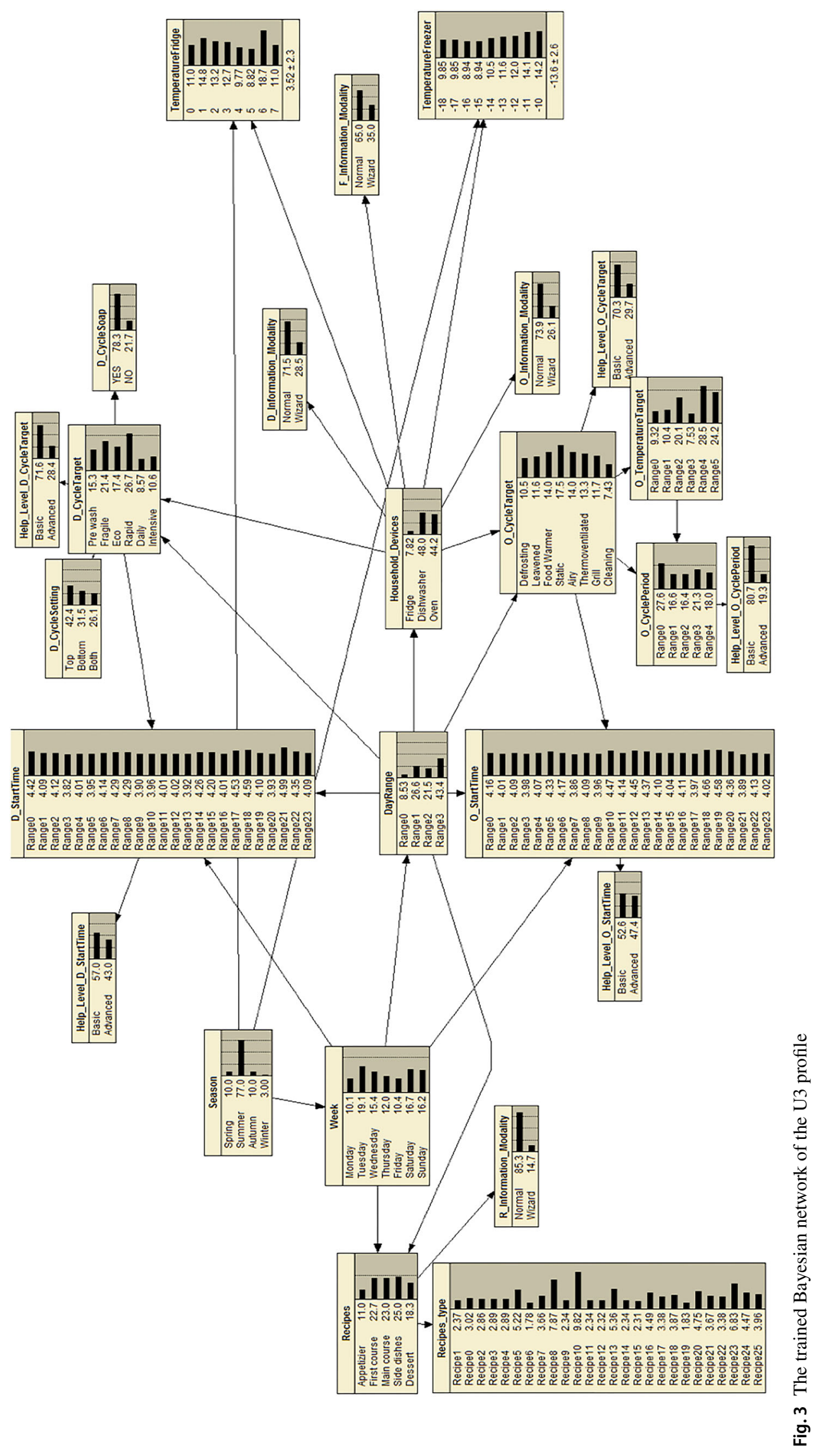




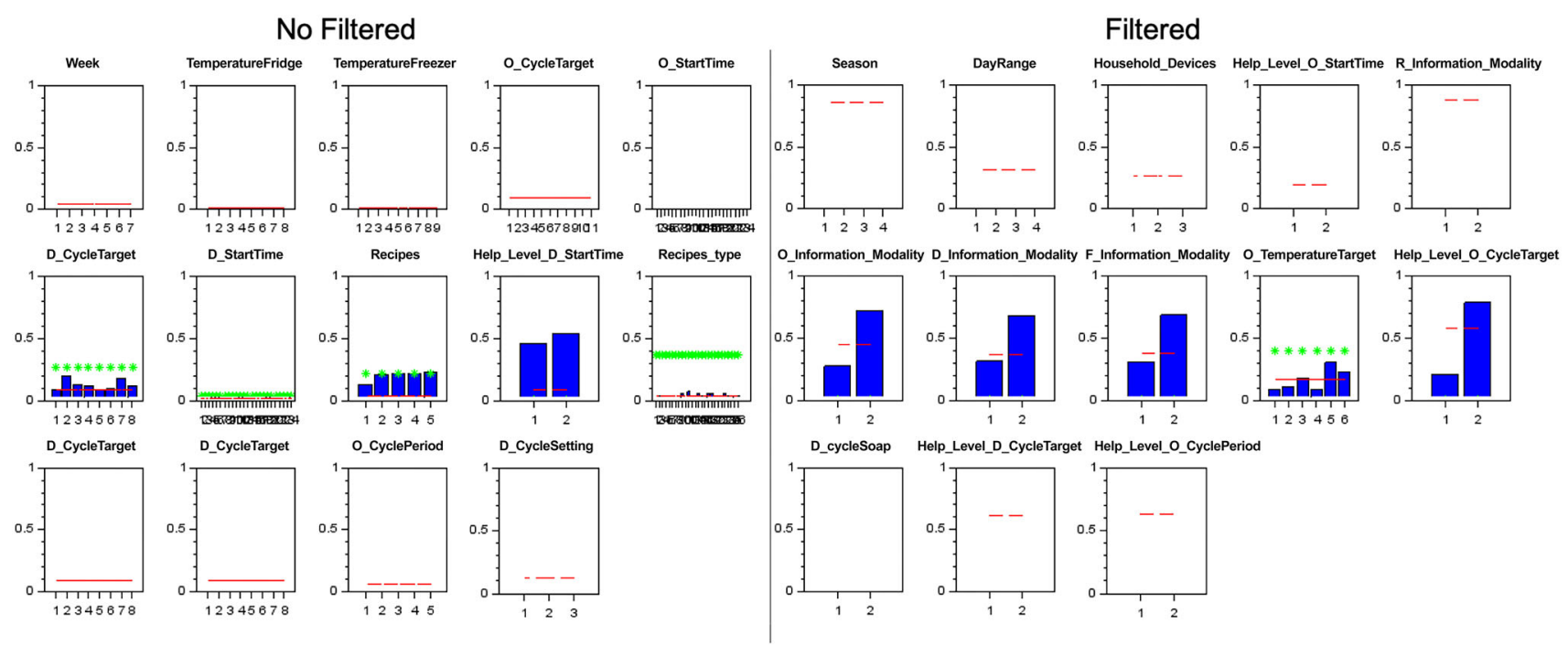

Fig. 4 The U3 BBN simulation

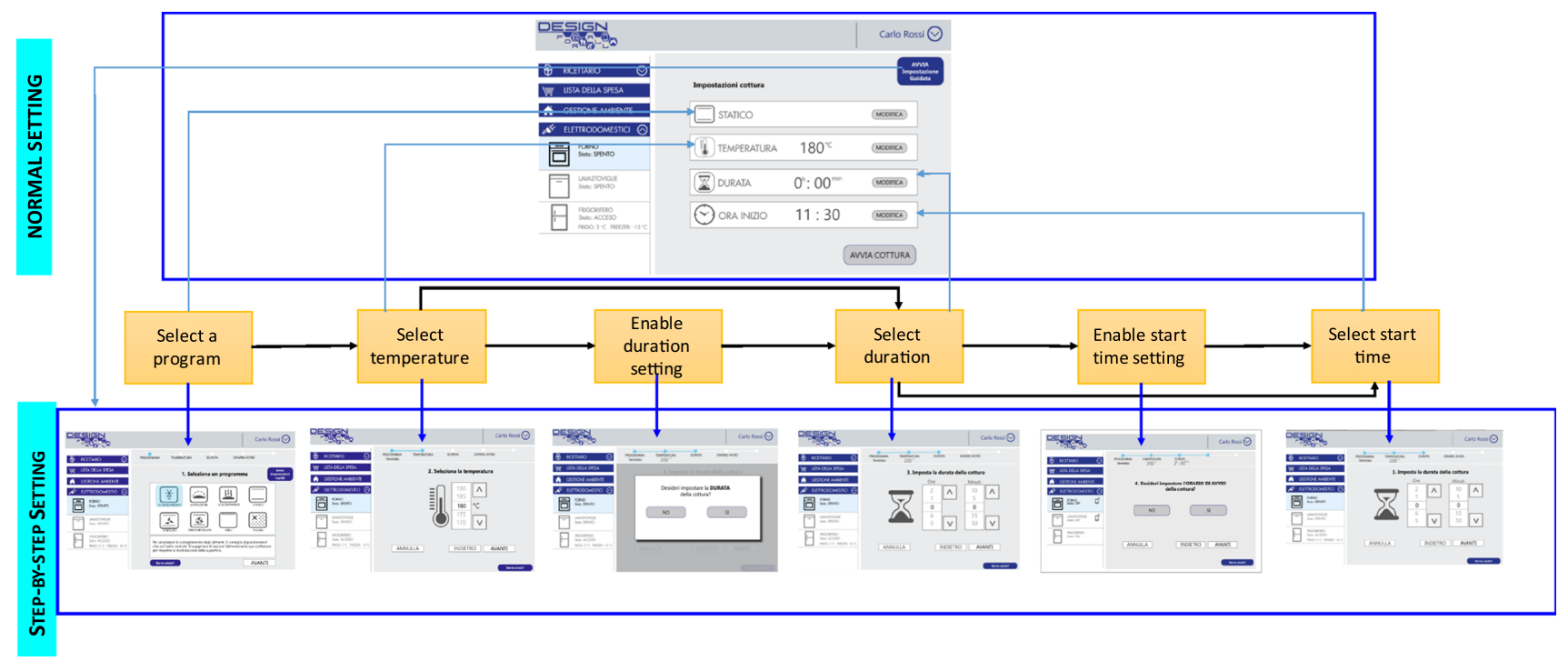

Fig. 5 Graphic user interface (on the top: normal; below: wizard)

ments such as color blindness, visual disturbances, motor problem. Using existing guidelines, the graphic features (e.g. color, text and buttons dimension, image size, etc.) and configurations associated with a specific disability have been designed.

Similarly, it has been done for the contents of the interface. The quantity and type of information that the interface gives to the user are designed on the user's abilities.

\section{Virtual reality environment}

In order to evaluate the Graphical User Interface (GUI) and the above presented system a VR interactive environment has been designed and developed.
The VR interactive environment has been used as main tool for the evaluation of the GUI with subjects instead of a real environment. The reasons are many. Firstly, the VR interactive environment is a flexible tool, which can be modified in order to reproduce and simulate different environments and situations. In particular, once created, a VR interactive environment can be easily edited, shared, visualized with different tools, and can be also enriched with additional items of information. All these activities have a limited cost if compared with similar ones performed using physical prototypes, which in this case are made of whole rooms including furniture, appliances, etc. Moreover, this environment and its components can be managed and controlled both with remote systems (such us, in this case, the GUI) and by the subjects during the evaluation sessions. Furthermore, the use of Vir- 
tual Prototypes has proven to be an effective tool to use for the evaluation of objects in other research areas, such as in the product development process, where it is used more and more [60-63].

Consequently, all the components that traditionally constitute an Italian kitchen have been identified, in order to consider their integration into the VR environment: undercounter furniture, with drawers or doors, and wall units, with hinged doors; built-in or free-standing home appliances: refrigerator, freezer, hob, oven, microwave, dishwasher, and washing machine and worktop.

The VR interactive environment was developed using the Unity3D software, a software tool largely used for the development of VR applications and used in the Virtual Environment laboratories of the University and the Institute involved. The VR environment supported the validation of the adaptive GUI with regard to the control of the household appliances and the control of the environment, both in the case of users without cognitive dysfunction (normal setup), and users with some cognitive dysfunction (wizard setup). In order to test the adaptive interface in the VR system the GUI and the VR environment are directly connected. In details, the adaptive GUI makes visible the inputs to the VR environment by using updated values of some control variables (as a result of events, such as clicking on the "next" button by the user), so that they can be read in a client/server system. Similarly, the VR environment makes visible the updated values of the variables (according to the events in the scene, such as "open the fridge door" or "illuminated shelves"), so that they can be read in a client/server system from the adaptive GUI. In this case through the Database the data about the users' profile (age, gender, technology attitude, possible cognitive dysfunction etc.) and the real home environment measured by sensors (brightness, temperature, etc.) are collected and analyzed in order to adapt the GUI characteristics. The interaction between the user and the VR environment only occurred through the GUI, due to the fact that the use of other devices for interacting with the environment could influence the evaluation of the GUI. In fact, most of the devices used for interacting with VR environments involves the use of one or both hands (gloves, remote controls, etc.), and can affect the validation of the touch screen application.

The virtual prototypes of the kitchen have been firstly developed by using various 3D modelling software tools (i.e. 3DStudioMax, Rhinoceros, Solidworks), depending on the characteristics of each following objects: the structural elements of the environment (floor, ceiling, walls, window, door, lights, etc.); home appliances (fridge, oven, dishwasher, hob and hood); furniture (under-counter furniture, shelves, table and chairs); accessories (plates, glasses, bowls, pots, cutlery, etc.); food items (some in the fridge, like eggs, cheese and vegetables, and others in the cabinets, like potatoes and oil).

Then, these have been imported into the Unity3D software using various interchange data formats. Therefore, they have been assembled in order to create the static VR kitchen environment. Consequently, materials and textures have been applied to it, and several lights have been installed into the kitchen, for simulating artificial lights, and outside it, for simulating the sun.

Finally, the three home appliances (refrigerator, oven and dishwasher) have been made dynamic, that is, manageable by the adaptive GUI (Fig. 6). Specifically, using the "home appliances" section of the adaptive interface, it is possible to individually select the refrigerator, the oven and the dishwasher and, for each one it is possible to set the program, the temperature, the delay start and time (Figs. 7, 8).

\section{System usability evaluation}

A preliminary usability evaluation test has been carried out to assess the system in terms of usability for each user target category (Fig. 9). This evaluation aimed to measure usability perceived by users during the first use of the smart system.

\subsection{Equipment and setting}

The adaptive GUI was presented on a Samsung Galaxy Tab A $\left(10.1^{\prime \prime}\right)$. The experiment took place in an immersive virtual reality system called CAVE. The CAVE is a projection room consisting of four screens placed in a mutual position on which the computer-generated environments are rearprojected and of different position sensors (trackers) applied for detecting patient's movements and transmit them to the computer, so that can modify the three-dimensional image according to the patient's point of view.

\subsection{Participants}

For the purpose of the study, a total of $\mathbf{6}$ participants were recruited in this observational study. To be enrolled, the following inclusion criteria were fixed:

- Age 50 and older;

- Previous experience in the use of touch-screen technologies.

- For Cognitive Impairment: Mini Mental State Examination (MMSE) score between 22 and 28 (early and mild impaired patients who retained the physical and functional ability to participate in a cognitive or motor stimulation program) (U1, U3). 


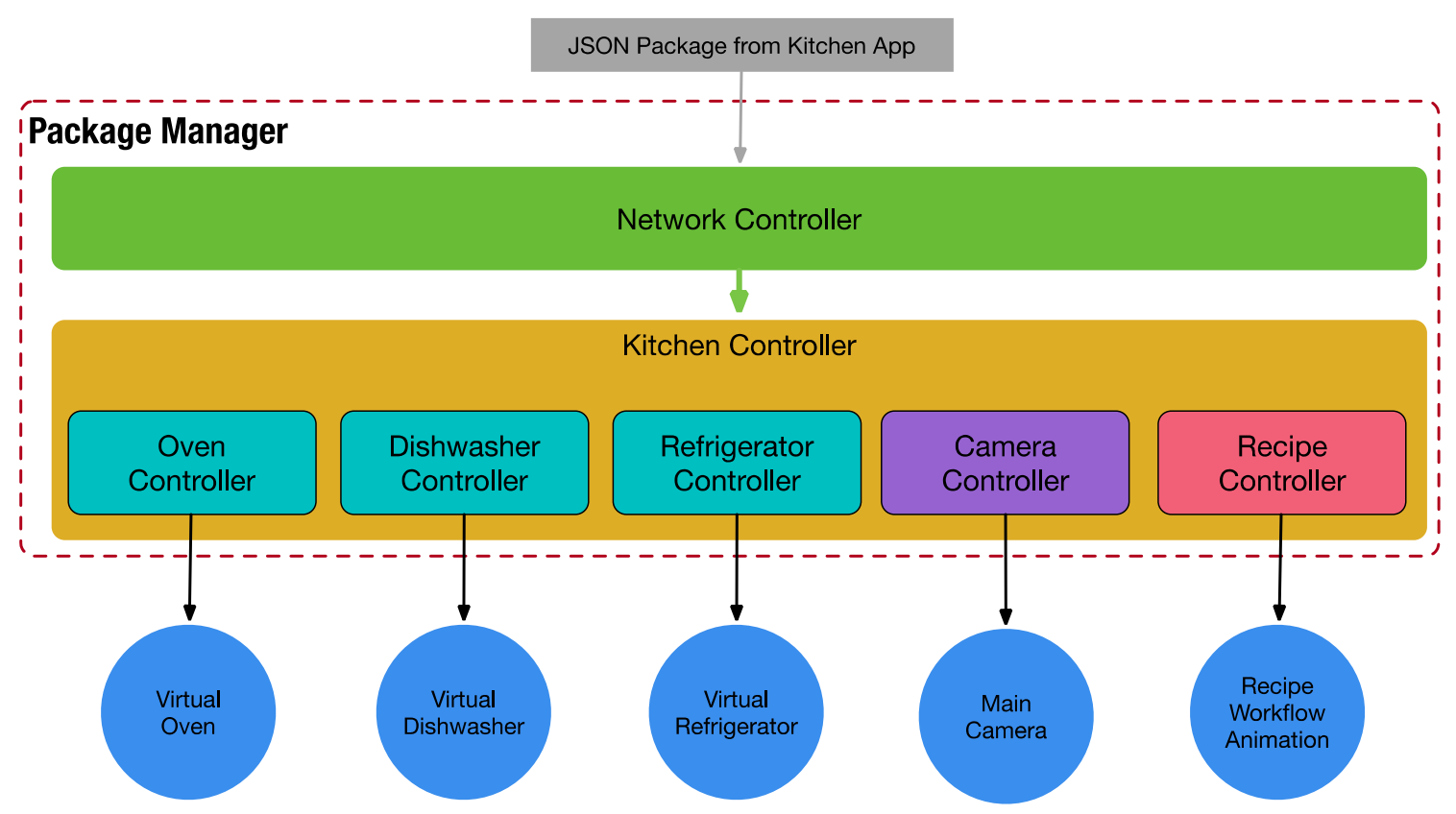

Fig. 6 Package manger of virtual reality environment and GUI communication

Fig. 7 Virtual prototypes of the home appliances and adaptive GUI

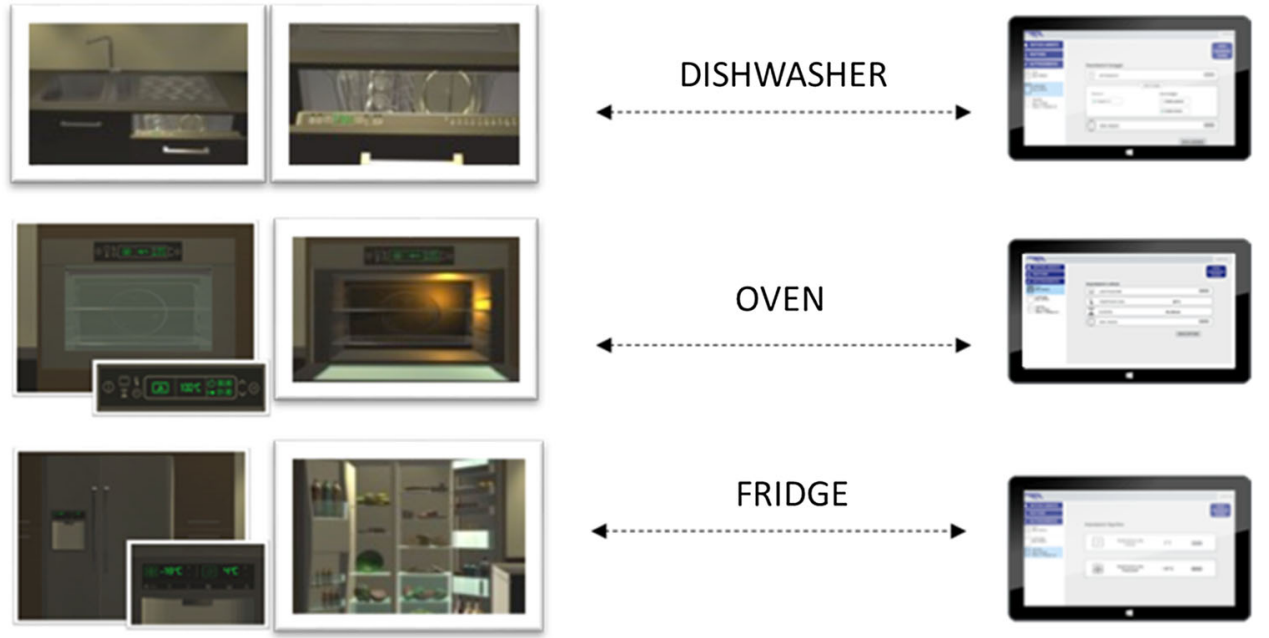

- For Dexterity Problem: Functional Dexterity Test score between 26 and 50 (Moderately and Minimally Functional) (U5, U6).

- For Visual Problem: Ranges of Vision Loss (based on visual acuity) score between Mild $(<0.8$ and $>0.3)$ and Moderate $(<0.3$ and $>0.125)$ Vision loss (U2, U4).

The total sample was composed of 3 males and 3 females, with a mean age of 60.33 years old $(S D \pm 6.15)$.

\subsection{Procedure}

Participants were welcomed in one of the laboratory facilities and were debriefed regarding the overall activity and goals. They read and signed the informed consent, the experimenter explicitly invited them to ask for any doubt at any time during the activity.

The experimenter then walked patients to the immersive reality system, CAVE, where she/he was illustrated how the experiment was set up, then patients received a hands-on explanation of how the adaptive GUI was structured and how to operate it. 
Fig. 8 The virtual reality environment

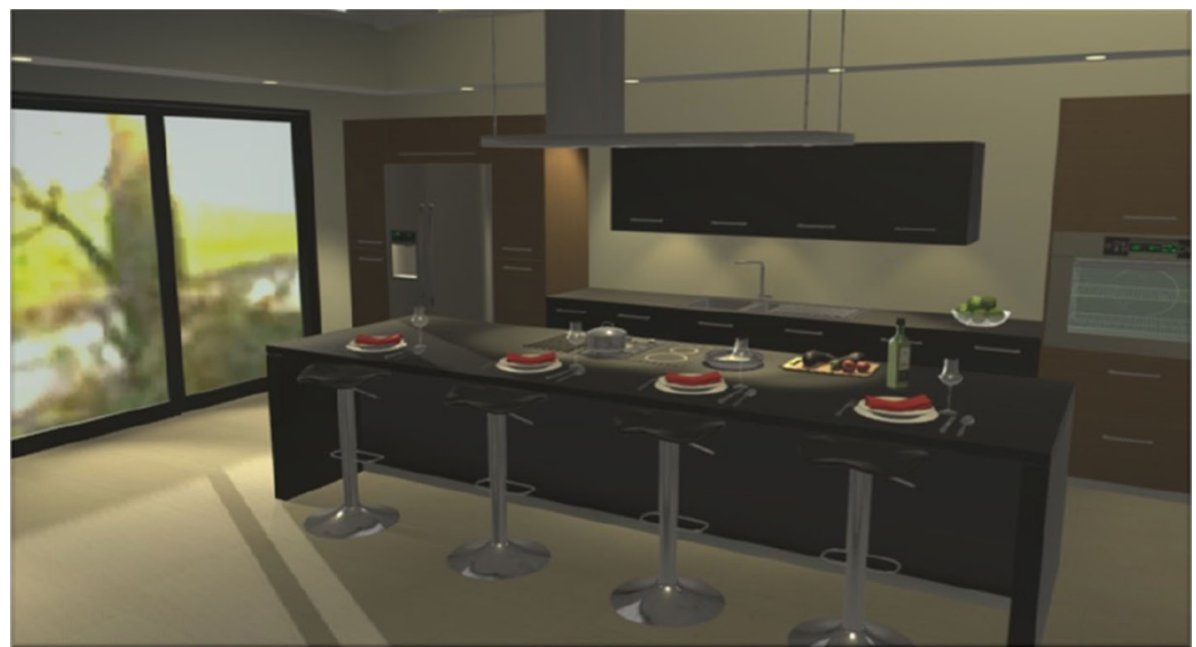

\section{System Usability Evaluation}

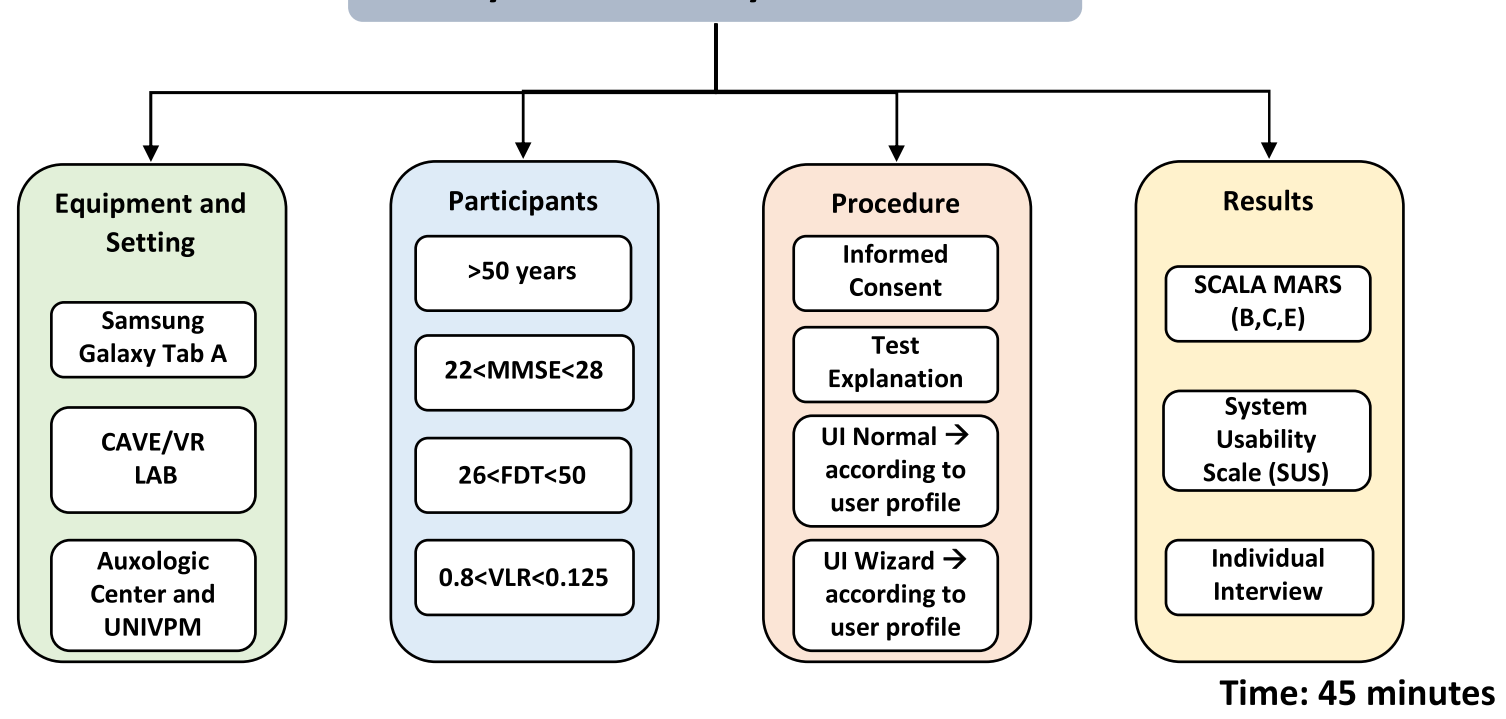

Fig. 9 System usability evaluation map

The participants in the research were exposed to simulations of everyday living environments ("virtual kitchen environment"), through the aforementioned systems of immersive virtual reality. During the session, the participants could move within the simulated environment carrying out tasks indicated by the research operator. Specifically, subjects were asked to interact with the GUI to control and manage some components of the kitchen, such as lightings and household appliances, and perform some activities, such as fill in a list of food items to be purchased, have suggestions and follow recipes. By using this approach, the authors kept the focus of the evaluation of the GUI, and not on the VR interactive environment, and on the interaction with it.
In detail, the system was presented to user and based on their profile and usage will provide the best UI mode (normal or wizard). At the end of the simulation, the participants filled out questionnaires that evaluated the user's experience in the interaction with the simulated interfaces (e.g. measuring the ease of use degree, learning difficulties, etc.). In detail the following surveys were carried out:

1. MARS scale (sections B, C, E);

2. System Usability Scale (SUS) which evaluates the usability of the interfaces;

3. Individual interview aimed at deeply examining in depth the dimensions of usability considered by the SUS questionnaire. 
The session lasted $45 \mathrm{~min}$.

\subsection{Results}

By analyzing the results, we can observe that the propose adaptive system can be considered usable for all considered user profile. Users performed a usability test of the Think Aloud Protocol based interface consisting of a sequence of 13 tasks designed in such a way as to test the main functionalities of the system. In detail, the System Usability Scale (SUS) questionnaire [64], calculated using the scores of all subjects, reports an average score of 70.42, which indicates a good level of usability of the system, as indicated in Fig. 10.

Furthermore, the Mobile Rating Scoring App (MARS) (sections B, C, E) provides an overall positive evaluation of the interface. The average score of sample responses to section $\mathrm{B}$, evaluating the functionality of the application, is $3 / 5$ indicating a sufficient level of functionality. The average score of the sample's answers to section $\mathrm{C}$, evaluating the application's aesthetics, is 3.23/5 indicating a discrete level of pleasantness. The average score of the sample responses to section E, evaluating the subjective quality of the application, is 2.79/5 indicating a sufficient level. Table 3 shows the score given by each participant both the SUS and MARS scale.

With respect to the patients with Cognitive Impairments, the usability test shows that the interface is sufficiently usable and adaptable, but the most commons problems are: comprehension and interaction with technology (scroll modality). The most of patients showed great difficulties in using tablet, in particular they struggle to scroll the pages and to search the correct program. This problem is transversal to the various tasks and is present for all the subjects. It can be easily solved by implementing a complete and interactive tutorial able to explain both the use of the tablet and the various functionalities of the interface itself. Alternatively, all the information could be entered on the same page, to the detriment of the images that for these patients were mainly confusing. Furthermore, the introduction of more visible buttons, and the insertion of some labels to clarify some commands would increase usability and error prevention. Table 4 summarizes, for the oven setting task, the problems that emerged from the two patients with Cognitive Deficit during the interview, the impact that these could have on usability, the solutions that could be put in place to solve these problems and the level of applicability of the same proposed by the experimenter.

With respect to the patients with Motor Deficit, the usability test shows that the interface is usable, and minor usability problems have been identified (Table 5). The most common problems are mainly due to the high proximity between buttons, which did not allow easy interaction with the tablet, causing errors such as selecting the wrong program or activating the help function. It has already been partially solved with the insertion of the adaptivity, but it can be further increased by the inserting white spaces to avoid the page overloaded. In addition, the zoom function accessible by double clicking on the tablet made the interaction with the technology problematic. For users with neuromotor deficit the zoom modality must be deactivated. Moreover, also in this case the introduction of a complete and interactive tutorial able to explain both the use of the tablet and the various functionalities of the interface itself has been requested by the users. Finally, during the interaction, one of the subjects has repeatedly reported the pleasure of the exercise, describing the tools as usable in a clear and intuitive way. He also appreciated what the practical aspects could be, highlighting how the application could be useful "not only for elderly people with difficulty in juggle in the kitchen, but also for young people, very skilled in technology but often heavily engaged in daily work activities".

Lastly, the interface has been evaluated with patients with Visual Impairment and a good system usability has emerged. Only few minor usability issues have been identified (Table 6) thanks to the UI adaptation with high contrast text and color inversion. The most common hurdle is due to the slight difficulty in understanding how to open the household appliances menu. It has already been solved by leaving the menu bar fixed and always displaying the household appliances links (i.e. oven, dishwasher and fridge). Finally, the introduction of more visible buttons (e.g. more color contrast with the text background color) and the introduction of a complete tutorial (to explain both the use of the tablet and the interface) would increase usability and error prevention.

\subsubsection{Heuristic evaluation}

A heuristic evaluation is a usability inspection method for computer software that helps to identify usability problems in the user interface design (UI) [65]. In particular, this evaluation was conducted using Jakob Nielsen's heuristics [66]: visibility of system status, match between system and the real world, user control and freedom, consistency and standards, error prevention, recognition rather than recall, flexibility and efficiency of use, aesthetic and minimalist design, help users recognize, diagnose, and recover from errors and lastly help and documentation.

In order to test the usability and the accessibility of the system, a team of five experts with the following profiles, has been recruited to conduct the evaluation: two geriatricians, two psychologists and an expert in human computer interaction. Results, which are reported in the Table 7, highlight that the propose solutions are suitable for all the considered profile (i.e. profile 1, cognitive impairment; profile 2, dexterity problems; profile 3, visually impaired).

Related to "Recognition rather than recall" heuristic, the same minor usability issue has been identified for Profile 2 and Profile 3: the knob, used for oven temperature and dura- 
Table 3 SUS and MARS individual scores

\begin{tabular}{|c|c|c|c|c|c|c|c|}
\hline & User 1 & User 2 & User 3 & User 4 & User 5 & User 6 & $\begin{array}{l}\text { Average } \\
\text { value }\end{array}$ \\
\hline SUS_1 & 3 & 3 & 3 & 2 & 4 & 3 & \\
\hline SUS_2 & 3 & 2 & 1 & 2 & 1 & 1 & \\
\hline SUS_3 & 3 & 4 & 4 & 4 & 4 & 5 & \\
\hline SUS_4 & 4 & 3 & 3 & 3 & 1 & 3 & \\
\hline SUS_5 & 4 & 5 & 2 & 2 & 4 & 4 & \\
\hline SUS_6 & 2 & 1 & 1 & 1 & 1 & 1 & \\
\hline SUS_7 & 3 & 5 & 3 & 3 & 3 & 4 & \\
\hline SUS_8 & 1 & 1 & 2 & 3 & 1 & 1 & \\
\hline SUS_9 & 3 & 4 & 5 & 5 & 4 & 4 & \\
\hline SUS_10 & 3 & 4 & 2 & 3 & 2 & 3 & \\
\hline SUS_TOT & 57.5 & 75 & 70 & 60 & 82.5 & 77.5 & 70.42 \\
\hline MARS_B6 & 2 & 4 & 2 & 3 & 4 & 4 & \\
\hline MARS_B7 & 2 & 4 & 1 & 3 & 3 & 4 & \\
\hline MARS_B8 & 2 & 4 & 1 & 3 & 2 & 3 & \\
\hline MARS_B9 & 3 & 4 & 2 & 4 & 4 & 4 & \\
\hline MARS_B_Mean & 2.25 & 4 & 1.5 & 3.25 & 3.25 & 3.75 & 2.75 \\
\hline MARS_C10 & 4 & 4 & 0 & 4 & 4 & 4 & \\
\hline MARS_C11 & 3 & 3 & 0 & 3 & 3 & 3 & \\
\hline MARS_C12 & 3 & 3 & 3 & 3 & 3 & 3 & \\
\hline MARS_C_Mean & 3.3 & 3.3 & 3 & 3.3 & 3.3 & 3.3 & 3.23 \\
\hline MARS_E_20 & 2 & 5 & 2 & 3 & 4 & 3 & \\
\hline MARS_E_21 & 3 & 5 & 2 & 2 & 3 & 3 & \\
\hline MARS_E_22 & 2 & 4 & 1 & 2 & 1 & 2 & \\
\hline MARS_E_23 & 3 & 5 & 3 & 1 & 3 & 3 & \\
\hline MARS_E_Mean & 2.5 & 4.75 & 2 & 2 & 2.75 & 2.75 & 2.79 \\
\hline
\end{tabular}

Table 4 Usability evaluation with cognitive impairments patients

\begin{tabular}{lllll}
\hline Task & Problems & Impact & Suggested solution & Solution feasibility \\
\hline Oven section & $\begin{array}{l}\text { Slight difficulty in understanding } \\
\text { how to select the section } \\
\text { Buttons too close }\end{array}$ & Medium & Insert tutorial & Medium applicable \\
$\begin{array}{l}\text { Set static program } \\
\text { Set } 200^{\circ} \mathrm{C} \text { temperature }\end{array}$ & - & Medium & Insert the label & Easily applicable \\
$\begin{array}{l}\text { Set } 2: 45 \text { duration time } \\
\text { Set the delay start }\end{array}$ & - & - & - & - \\
& $\begin{array}{c}\text { Difficulty in understanding the } \\
\text { delay start setting }\end{array}$ & Medium & Insert tutorial/help & Easily applicable \\
Start the cooking & $\begin{array}{c}\text { The start cooking button is not } \\
\text { detected immediately }\end{array}$ & Low & $\begin{array}{c}\text { Move to the top most key } \\
\text { and highlight it }\end{array}$ & Easily applicable \\
\hline
\end{tabular}

Table 5 Usability evaluation with dexterity problems patients

\begin{tabular}{lllll}
\hline Task & Problems & Impact & Suggested solution & Solution feasibility \\
\hline Oven section & - & - & - & - \\
Set static program & Buttons too close & Medium & Insert the label & Easily applicable \\
Set $200^{\circ} \mathrm{C}$ temperature & Too small icons & - & Resize the icons size & Easily applicable \\
Set $2: 45$ duration time & - & - & - & - \\
Start the cooking & The start cooking button is not & Low & Move to the top most key & Easily applicable \\
& detected immediately & & and highlight it & \\
\hline
\end{tabular}


Fig. 10 The system usability scale (SUS) score

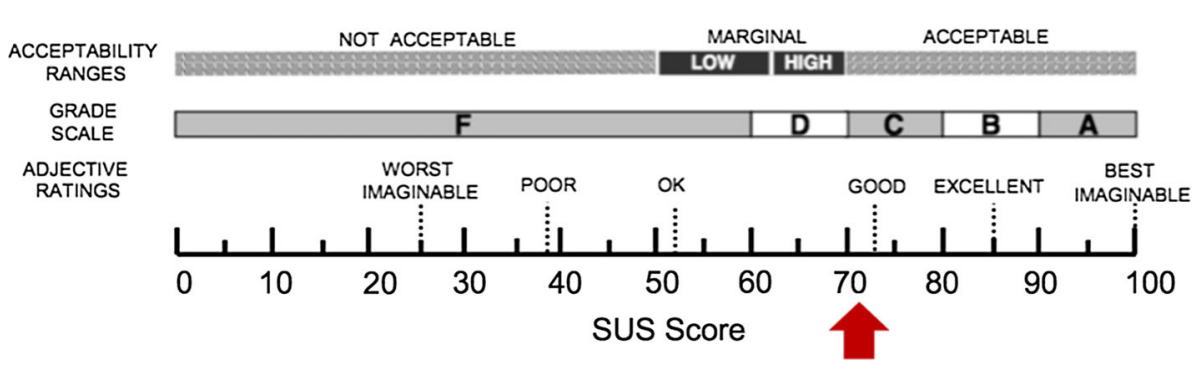

Table 6 Usability evaluation with visual deficit patients

\begin{tabular}{lllll}
\hline Task & Problems & Impact & Suggested solution & Solution feasibility \\
\hline Oven section & $\begin{array}{l}\text { Slight difficulty in opening the } \\
\text { appliance menu }\end{array}$ & Low & Fix the drop-down menu & Medium applicable \\
Set static program & - & - & - & - \\
Set $200^{\circ} \mathrm{C}$ temperature & - & - & - & - \\
Set $2: 45$ duration time & - & - & - & - \\
Start the cooking & $\begin{array}{c}\text { The start cooking button is not } \\
\text { detected immediately }\end{array}$ & Low & Move to the top most key & Easily applicable \\
& & & and highlight it
\end{tabular}

tion changing, may not be intuitive for an inexpert user. Such problem may be solved by adopting a different interaction modality. In addition, for Profile 1 a relevant usability issue has been detected, as the amount of information about each program are probably too much to be correctly manage: this can be recover improving the wizard.

\section{Discussion and conclusion}

Despite the interesting results collected, the sample size of the study is relatively small, and this does not allow a proper generalization of the data. For this reason, it is important to take into account the system usability evaluation as a pilot, with the aim of involving a higher number of users during the next research activities. As demonstrated by the SUS and MARS scores, the interface is generally usable by users with different impairments, in terms of typology and severity.

Collecting the opinions of the subjects after the experience in the CAVE it emerges that the subjects consider the task complex, but mainly because they do not think to have the skills needed to use this technology (tablet). For this reason, they indicate as main target subjects younger than themselves, at least from the generation of their children. The need to provide a user-friendly tutorial, before starting the use of technology, clearly emerged to prevent patients from feeling frustrated by the difficulties, which negatively interfere with the ease to perform tasks.

The execution of several tasks allows obtaining interesting outcomes from different perspectives: usability and adaptability. From one hand, each task allows observing specific usability problems; from the other one, the overall test allows taking into account previous interactions and evaluating the improvement on usability due to the system adaptation.

During the usability evaluation, some technical problems hindered the perfect outcome of the task and that could have diminished the perception of the usability of the application. They mainly refer to minor usability problems such as make some buttons more visible, insert some labels to clarify some commands, and rewrite some sentences to improve their understandability.

The major usability problem concerns the error prevention and the recovery from errors. Several feasible solutions were hypnotized and will be implemented to solve it. For example, a user passed several times to the full version of the recipe accidentally. To remedy this error, the experimenter needed to prevent the animations from going out of sync.

A couple of tasks were not completed by one of the two patients with dexterity problems. Some adaptations related to the pages layout (e.g. buttons closeness) allowed to the user to complete the following tasks, but some difficulties remained (e.g. double tap zoom). This suggested to modify the interaction modalities to simply the navigation anyway.

In case of users with cognitive impairments, the benefits of system adaptation (i.e. shift to wizard modality) were fully appreciated and were reflected by users' performance in following tasks. However, transversal problems such as the use of the scroll function should be necessarily faced to improve the user experience of these users.

UI adaptations in terms of high contrast text, color inversion and zoom allows the successfully execution of tasks by users with visual impairments.

In conclusion, the analysis gave several feedbacks to improve the static adaptive behavior of the system. On the 


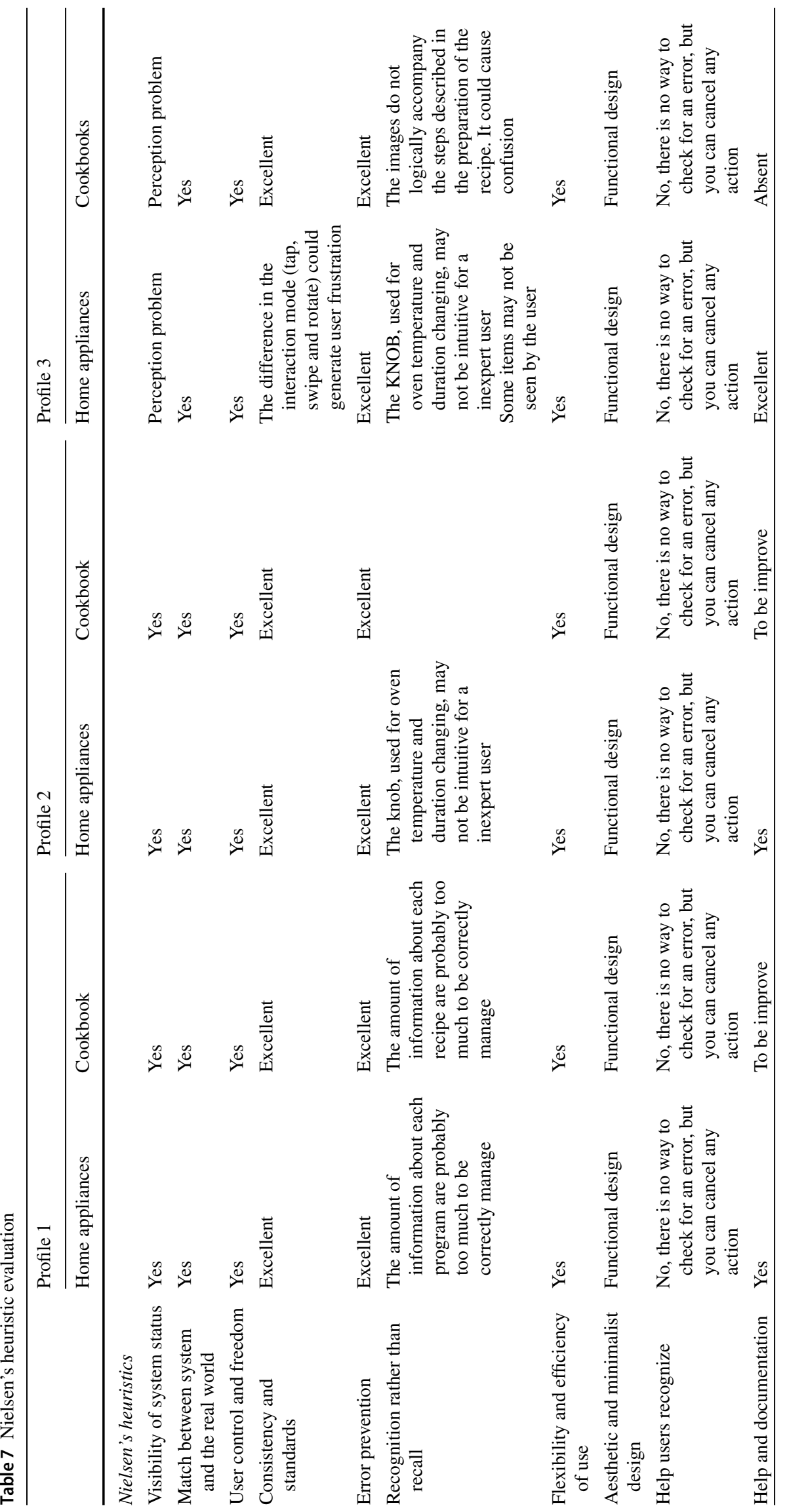


other hand, a further analysis should be performed to investigate the dynamic adaptive behavior. In the next future, apposite scenario and test procedure will be defined and implemented to face this issue. Then, a trial test will be carry out through a real environment installed at user's sample homes.

Acknowledgements The present research study was partially funded by the project "D4All: Design for all" (CTN01_00128_297089), by the Italian Minister of University and Research, under the National Cluster Ambient Assisting Living Technologies (TAV).

\section{References}

1. Cook, D., Das, S.K.: Smart Environments: Technology, Protocols and Applications, vol. 43. Wiley, Hoboken (2004)

2. World Health Organization: World Report on Disability. World Health Organization, Geneva (2011)

3. World Health Organization: Global Health and Aging. World Health Organization, Geneva (2011)

4. Plan, A.: Communication from the Commission to the European Parliament, the Council, the European Economic and Social Committee and the Committee of the Regions. European Commission, Brussels (2011)

5. Van den Broek, G., Cavallo, F., Wehrmann, C. (eds.): AALIANCE Ambient Assisted Living Roadmap, vol. 6. IOS Press, Amsterdam (2010)

6. Huch, M., Kameas, A., Maitland, J., McCullagh, P., Roberts, J., Sixsmith, A., Wichert, R.: Handbook of ambient assisted living. In: Augusto, J.C. (ed.). IOS Press, Amsterdam (2012)

7. Gullà, F., Ceccacci, S., Menghi, R., Germani, M.: An adaptive smart system to foster disabled and elderly people in kitchen-related task. In: Proceedings of the 9th ACM International Conference on PErvasive Technologies Related to Assistive Environments, p. 27. ACM (2016)

8. Chaminda, H.T., Klyuev, V., Naruse, K.: A smart reminder system for complex human activities. In: 14th International Conference on Advanced Communication Technology (ICACT), 2012, pp. 235-240. IEEE (2012)

9. Sponselee, A.A.G.: Acceptance and Effectiveness of Telecare Services from the End-User Perspective. Eindhoven University of Technology, Eindhoven (2013)

10. DIS, I.: 9241-210:2010. Ergonomics of Human System InteractionPart 210: Human-Centred Design for Interactive Systems. International Standardization Organization (ISO), Geneva (2009)

11. Jiménez-Mixco, V., Villalar González, J.L., Arca, A., CabreraUmpierrez, M.F., Arredondo, M.T., Manchado, P., García-Robledo, M.: Application of virtual reality technologies in rapid development and assessment of ambient assisted living environments. In: Proceedings of the 1st ACM SIGMM International Workshop on Media Studies and Implementations that Help Improving Access to Disabled Users, pp. 7-12. ACM (2009)

12. Hall, T.W., Navvab, M., Maslowski, E., Petty, S.: Virtual reality as a surrogate sensory environment. In: Advances in Robotics and Virtual Reality, pp. 251-273. Springer, Berlin (2012)

13. Rothrock, L., Koubek, R., Fuchs, F., Haas, M., Salvendy, G.: Review and reappraisal of adaptive interfaces: toward biologically inspired paradigms. Theor. Issues Ergon. Sci. 3(1), 47-84 (2002)

14. Benyon, D., Murray, D.: Applying user modeling to human-computer interaction design. Artif. Intell. Rev. 7(3), 199-225 (1993)

15. Letsu-Dake, E., Ntuen, C.A.: A case study of experimental evaluation of adaptive interfaces. Int. J. Ind. Ergon. 40(1), 34-40 (2010)
16. Grundy, J., Hosking, J.: Developing adaptable user interfaces for component-based systems. Interact. Comput. 14(3), 175-194 (2002)

17. Schneider-Hufschmidt, M., Malinowski, U., Kuhme, T.: Adaptive User Interfaces: Principles and Practice. Elsevier Science Inc., Amsterdam (1993)

18. Shneiderman, B., Maes, P.: Direct Manipulation vs. Interface Agents. Interactions 4(6), 42-61 (1997)

19. Langley, P.: User modeling in adaptive interface. In: UM99 User Modeling, pp. 357-370. Springer, Vienna (1999)

20. Fischer, G.: User modeling in human-computer interaction. User Model. User Adapt. Interact. 11(1), 65-86 (2001)

21. Ghédira, C., Maret, P., Fayn, J., Rubel, P.: Adaptive user interface customization through browsing knowledge capitalization. Int. J. Med. Inform. 68(1), 219-228 (2002)

22. Bental, D., Cawsey, A., Pearson, J., Jones, R.: Adapting web-based information to the needs of patients with cancer. In: Adaptive Hypermedia and Adaptive Web-Based Systems, pp. 27-37. Springer, Berlin (2000)

23. Shakshuki, E.M., Reid, M., Sheltami, T.R.: An adaptive user interface in healthcare. Procedia Comput. Sci. 56, 49-58 (2015)

24. Vasilyeva, E., Pechenizkiy, M., Puuronen, S.: Towards the framework of adaptive user interfaces for eHealth. In: Proceedings of 18th IEEE Symposium on Computer-Based Medical Systems, 2005, pp. 139-144. IEEE (2005)

25. Song, I.J., Cho, S.B.: Bayesian and behavior networks for contextadaptive user interface in a ubiquitous home environment. Expert Syst. Appl. 40(5), 1827-1838 (2013)

26. Namgoong, H., Sohn, J.C., Cho, Y.J., Chung, Y.K.: An adaptive user interface in smart environment exploiting semantic descriptions. In: IEEE 10th International Symposium on Consumer Electronics, 2006, ISCE'06, pp. 1-6. IEEE (2006)

27. Yen, G.G., Acay, D.: Adaptive user interfaces in complex supervisory tasks. ISA Trans. 48(2), 196-205 (2009)

28. El-Bakry, H.M., Riad, A.M., Abu-Elsoud, M., Mohamed, S., Hassan, A.E., Kandel, M.S., Mastorakis, N.: Adaptive user interface for web applications. In: Recent Advances in Business Administration: Proceedings of the 4th WSEAS International Conference on Business Administration (ICBA'10), pp. 20-22 (2010)

29. Rebai, R., Maalej, M.A., Mahfoudhi, A., Abid, M. : Building and evaluating an adaptive user interface using a Bayesian network approach. Int. J. Comput. Sci. Inf. Secur. (IJCSIS) 14(7), 548-565 (2016)

30. Viano, G., Parodi, A., Alty, J., Khalil, C., Angulo, I., Biglino, D., Lachaud, P.: Adaptive user interface for process control based on multi-agent approach. In: Proceedings of the Working Conference on Advanced Visual Interfaces, pp. 201-204. ACM (2000)

31. Bongartz, S., Jin, Y., Paternò, F., Rett, J., Santoro, C., Spano, L.: Adaptive user interfaces for smart environments with the support of model-based languages. Ambient Intell. 33-48 (2012)

32. Mitrovic, N., Mena, E.: Adaptive user interface for mobile devices. Lect. Notes Comput. Sci. 2545, 29-43 (2002)

33. Nivethika, M., Vithiya, I., Anntharshika, S., Deegalla, S.: Personalized and adaptive user interface framework for mobile application. In: 2013 International Conference on Advances in Computing, Communications and Informatics (ICACCI), pp. 1913-1918. IEEE (2013)

34. Wang, X., Hua, Q.Y., Zou, F., Guo, L.: An adaptive user interface model for mobile devices based on perceptual control theory. In: 5th IEEE International Conference on Software Engineering and Service Science (ICSESS), 2014, pp. 908-911. IEEE (2014)

35. Park, J., Han, S.H., Park, Y.S., Cho, Y.: Adaptable versus adaptive menus on the desktop: performance and user satisfaction. Int. J. Ind. Ergon. 37(8), 675-684 (2007) 
36. Park, J., Han, S.H.: Complementary menus: combining adaptable and adaptive approaches for menu interface. Int. J. Ind. Ergon. 41(3), 305-316 (2011)

37. Plessas, A., Stefanis, V., Komninos, A., Garofalakis, J.: Field evaluation of context aware adaptive interfaces for efficient mobile contact retrieval. Pervasive Mob. Comput. 35, 51-64 (2017)

38. Brusilovsky, P., Eklund, J.: A study of user model based link annotation in educational hypermedia. J. Univers. Comput. Sci. 4(4), 429-448 (1998)

39. Brusilovsky, P., Pesin, L.: Adaptive navigation support in educational hypermedia: an evaluation of the ISIS-Tutor. CIT. J. Comput. Inf. Technol. 6(1), 27-38 (1998)

40. Greenberg, S., Witten, I.H.: Adaptive personalized interfaces-a question of viability. Behav. Inf. Technol. 4(1), 31-45 (1985)

41. Liu, J., Wong, C.K., Hui, K.K.: An adaptive user interface based on personalized learning. IEEE Intell. Syst. 18(2), 52-57 (2003)

42. Findlater, L., McGrenere, J.: A comparison of static, adaptive, and adaptable menus. In: Proceedings of the SIGCHI Conference on Human Factors in Computing Systems, pp. 89-96. ACM (2004)

43. Paramythis, A., Totter, A., Stephanidis, C.: A modular approach to the evaluation of adaptive user interfaces. In: Empirical Evaluation of Adaptive Systems. Proceedings of Workshop at the 8th International Conference on User Modeling, UM2001, pp. 9-24 (2001)

44. Gajos, K.Z., Czerwinski, M., Tan, D.S., Weld, D.S.: Exploring the design space for adaptive graphical user interfaces. In: Proceedings of the Working Conference on Advanced Visual Interfaces, pp. 201-208. ACM (2006)

45. Zudilova-Seinstra, E.: On the role of individual human abilities in the design of adaptive user interfaces for scientific problem solving environments. Knowl. Inf. Syst. 13(2), 243-270 (2007)

46. Gajos, K.Z., Everitt, K., Tan, D.S., Czerwinski, M., Weld, D.S.: Predictability and accuracy in adaptive user interfaces. In: Proceedings of the SIGCHI Conference on Human Factors in Computing Systems, pp. 1271-1274. ACM (2008)

47. Lin, Y., Breugelmans, J., Iversen, M., Schmidt, D.: An adaptive interface design (AID) for enhanced computer accessibility and rehabilitation. Int. J. Hum. Comput. Stud. 98, 14-23 (2017)

48. Vaughan, N., Gabrys, B., Dubey, V.N.: An overview of selfadaptive technologies within virtual reality training. Comput. Sci. Rev. 22, 65-87 (2016)

49. Huang, X., Naghdy, F., Naghdy, G., Du, H., Todd, C.: The combined effects of adaptive control and virtual reality on robot-assisted fine hand motion rehabilitation in chronic stroke patients: a case study. J. Stroke Cere Brovasc. Dis. 27, 221-228 (2017)

50. Matsas, E., Vosniakos, G.C., Batras, D.: Prototyping proactive and adaptive techniques for human-robot collaboration in manufacturing using virtual reality. Robot. Comput. Integr. Manuf. 50, $168-180$ (2017)

51. Maurya, S., Arai, K., Moriya, K., Arrighi, P.A., Mougenot, C.: A mixed reality tool for end-users participation in early creative design tasks. Int. J. Interact. Des. Manuf. (IJIDeM) 1-20 (2018)
52. Cooper, A.: The inmates are running the asylum: why high-tech products drive us crazy and how to restore the sanity. SAMS, Indianapolis (1999)

53. Gulliksen, J., Göransson, B., Boivie, I., Blomkvist, S., Persson, J., Cajander, Å.: Key principles for user-centred systems design. Behav. Inf. Technol. 22(6), 397-409 (2003)

54. Gullà, F., Ceccacci, S., Menghi, R., Cavalieri, L., Germani, M.: Adaptive interface for smart home: a new design approach. In: Italian Forum of Ambient Assisted Living, pp. 107-115. Springer, Cham (2016)

55. Brewer, J., Dardailler, D.: World Wide Web Consortium (W3C) Web Accessibility Initiative (WAI). World Wide Web Consortium, Cambridge (2000)

56. Pearl, J.: Probabilistic Reasoning in Intelligent Systems: Networks of Plausible Inference. Morgan Kaufmann, Burlington (2014)

57. Nyberg, J.B., Marcot, B.G., Sulyma, R.: Using Bayesian belief networks in adaptive management. Can. J. For. Res. 36(12), 3104-3116 (2006)

58. Gullà, F., Cavalieri, L., Ceccacci, S., Germani, M.: A BBN-based method to manage adaptive behavior of a smart user interface. Procedia CIRP 50, 535-540 (2016)

59. W3C., W. C.: http://www.w3.org/TR/WCAG20/ (2017). Accessed 24 May 2017

60. Wang, G.: Definition and review of virtual prototyping. J. Comput. Inf. Sci. Eng. 2(3), 232-236 (2002)

61. Carulli, M., Bordegoni, M., Cugini, U.: An approach for capturing the voice of the customer based on virtual prototyping. J. Intell. Manuf. (2012). https://doi.org/10.1007/s10845-012-0662-5

62. Caruso, G., Re, G.M., Carulli, M., Bordegoni, M.: Novel augmented reality system for contract design sector. Comput. Aided Des. Appl. 11(4), 389-398 (2014). https://doi.org/10.1080/ 16864360.2014.881181

63. Bordegoni, M., Carulli, M.: Evaluating industrial products in an innovative visual-olfactory environment. J. Comput. Inf. Sci. Eng. 16(3), 030904 (2016). https://doi.org/10.1115/1.4033229

64. Sauro, J.: A practical guide to the system usability scale: background, benchmarks \& best practices. Measuring Usability LLC, Denver (2011)

65. Nielsen, J., Molich, R.: Heuristic evaluation of user interfaces. In: Proceedings of the SIGCHI Conference on Human Factors in Computing Systems, pp. 249-256. ACM (1990)

66. Nielsen, J.: Heuristic evaluation. In: Nielsen, J., Mack, R.L. (eds.) Usability Inspection Methods. Wiley, New York (1994)

Publisher's Note Springer Nature remains neutral with regard to jurisdictional claims in published maps and institutional affiliations. 